Most regions in New York State furnish illustration of all the causes and results of weathering and erosion, of the various phenomena connected with young and mature drainage, of shore features as exhibited in lakes or ponds and of glaciation. I find it possible to have my pupils study in the field every topic under the land and land waters except mountains and volcanic action. The places most worthy of examination and giving the richest results will probably be stream valleys, quarries and other places where the rock is exposed, roadsides and excavations of earth, and the shores of ponds or lakes.

\title{
THE PRESENTATION OF THE IDEAS OF POSITIVE AND NEGATIVE NUMBER TO BEGINNERS.
}

\section{Roв'T M. McDiLL. \\ New Castle, Indiana.}

If we can show the need for negative number and even a few of its applications, the subject will be much simplified. The teacher will be compelled to consider four questions: What are the facts with regards to negative number? What material does the pupil have in mind, or easily in reach, which will enable him to grasp negative number? What particular questions or devices will help to bring out the laws which govern its use? Also wherein do mathematicians disagree with regard to negative number?

The class may be told that in arithmetic they have dealt with number from zero up to an indefinitely large number, while in algebra he must study the system from an indefinitely large (or as he will later call it small) negative quantity up through zero to an indefinitely large number. At the same time the teacher may have a line on the blackboard; this line may be marked off into units numbered both ways from zero, those below or to the left being marked minus. In this way the pupil is shown that zero may represent a starting place and not necessarily absolutely nothing.

The thermometer is always at hand in the school room and it furnishes probably the best illustration which the teacher can use for the first lesson. The pupil will be reminded that in the weather reports $10^{\circ}$ means $10^{\circ}$ above zero, while $-10^{\circ}$ means $10^{\circ}$ below zero. A large percent of the class will be familiar with 
this use of the minus sign. And even those not familar with the weather reports very quickly take to this method of representing temperatures above zero by plus and temperatures below by over the custom adopted by the weather bureau of representing temperatures above zero by plus and temperatures below by minus. And yet in this simple and natural illustration we have + and - representing not operations but qualities. While the pupils are looking at the thermometer and thinking of the weather reports, ask some one to subtract 8 from 6 . He will look at you in amazement and say that it is not possible to stibtract 8 from 6. Ask him if it is possible to become $8^{\circ}$ colder than $+6^{\circ}$ he will tell you that $-2^{\circ}$ would be $8^{\circ}$ colder than $+6^{\circ}$. Tell him that this is what you mean by subtracting $8^{\circ}$ from $6^{\circ}$ and you have opened up a new field. After several such questions have been asked and the pupils have come to answer readily ask a pupil to add -8 and 12 . If he is confused turn to your line on the blackboard and with him count up to $\mathrm{I} 2 \mathrm{units}$ from the point -8 . You have gone a long way toward the answer when you have made the pupil see the meaning of the question. Several years ago I heard a university professor, now a well known university president, say in a talk on imaginary numbers : "Why, they are misnamed! They are not imaginary at all. They are just as real as any numbers." And so they were to him. He knew how they are obtained and how to interpret and represent them. So if we can make real to the pupils these simple numerical problems we have gone a long way toward the development of the laws which govern their use.

The pupil may be told that in the future work distance to the right of the starting point is called + and he will anticipate that distance to the left is called -. Tell him that distance down is called minus and he will anticipate that distance up is called + . In this way, one of the fundamental conceptions of analytical geometry is awakened in his mind. My subject, as originally assigned me, is the presentation of positive and negative quantity. But in the development of the laws of signs quantity must represent number, - $a$ multiplied by $b$ can have absolutely no meaning to the beginner until he thinks of these letters representing numbers. It is one of the ideals of algebra to make ideas, conceptions, principles and proofs general. But it is also true that we get general ideas from particulars. The mind works from particulars to general conclusions, and then on to particular again. 
Therefore in the development of the laws of signs, with pupils who are really beginners, it will be necessary to use numbers for illustrations. This is especially true of addition and multiplcation.

There are four cases in addition, the addition of two positive numbers, the addition of a positive and a negative number, the addition of a negative and a positive number and the addition of two negative numbers. For practical purposes the first two of these cases may be ignored. The first because it has been treated in arithmetic and the second because from the nature of addition it is the same as the third.

The addition of a negative and a positive number may be illusstrated by the blackboard line already described. Find the point represented by the negative number and count up as many units as there are in the positive number. Write each problem together with its answer on the blackboard. As soon as the pupils are able to add readily having before them the line marked off into units, call attention to the fact that each result is the same as would be obtained by subtracting the less absolute value from the greater and prefixing the sign of the greater absolute value. The problem is thus reduced to one of arithmetical subtraction. If pupils have trouble in remembering which sign belongs to the result, the teacher may suggest that they let majorities rule. The fourth case, the addition of two negative numbers may be well illustrated by the money illustration or by the one of gains and losses. The addition of -5 and - Io may be compared to the combining of a debt of $\$ 5$ and a debt of $\$ 10$. The pupil will readily say a debt of $\$ \mathrm{I}_{5}$. And the fact that it is a debt may be indicated by prefixing the minus sign. Write many illustrations on the board, then call attention to the fact that in each case the result may be obtained by adding the absolute values of the numbers and prefixing the negative sign, to the result.

Then have the pupils commit the rules which may be worded as follows. To add a positive and a negative number, subtract the less absolute value from the greater and prefix the sign of the greater. Also, to add two negative numbers, add their absolute. values and prefix the negative sign.

After the rules have been learned, let them be freely used. It is not necessary to have in mind an illustration every time two quantities are added. Although it is desirable to be able to give one if called upon. It is a mistake to think that the mind must 
continually keep in conscious view every principle used. Each one of us uses freely the proposition, that "in the same or equal circles the greater of the two chords subtends the greater minor arc." We use this proposition even when its.proof is not before the conscious mind. I do not mean that we could not prove the proposition if called upon, but I do mean that we frequently use the conclusion without thinking through the proof. So in adding $\rightarrow 7$ and ro we do not always have in mind an illustration but we go ahead and use the established rule. What shall be done with the pupil who makes a blunder? Give him an illustration to bring out the blunder or have him review the rule? Probably he will need both treatments.

The subject of addition must not be left until it is thoroughly mastered, this is especially necessary because the problems in subtraction are so easily confused with those involving the addition of negative number. Then subtraction may be taken up. This will divide itself into two cases: The subtraction of a positive number and the subtraction of a negative number. The subtraction of a positive number may be illustrated as follows; on the line represented by the number system find the point represented by the minuend and then count down as many units as there are units in the subtrahend. Each problem may be written on the board and after several have been written show by parallel problems that subtracting a positive number is the same as adding a numerically equal negative number.

The subtraction of a negative number has often been compared to the taking away of a debt. The taking away of a debt of $\$ 5$ has the same effect on a man's financial standing as the giving of $\$ 5$ to him. And combining the two cases subtracting any number is the same as adding a numerically equal number with the sign changed.

Multiplication will divide itself into four cases. Expressed in abbreviated form by $\mathrm{a}+$ by $\mathrm{a}+; \mathrm{a}-$ by $\mathrm{a}+$; $\mathrm{a}+$ by $\mathrm{a}-$ and $\mathbf{a}-$ by $\mathbf{a}-$. The first need not be considered. The second may be illustrated by such problems as, What is five times a debt of $\$ 6$ ? That is $-\$ 6$ multiplied by 5 ? The pupil will readily say a debt of $\$ 30$. And how can the result be written to show that the result is a debt? On one morning the thermometer stood at $3^{\circ}$ below zero and the next morning it was four times as much below zero as the first. How cold was it the second morning? What is $-3^{\circ}$ times 4 ? After a series of different numbers are substi- 
tuted in the above questions the pupil is ready to conclude that a negative number multiplied by a positive number is negative. It is not easy to get good illustrations of a positive number multiplied by a negative number. There are two things that may be done. We may say that so far as abstract numbers are concerned, it makes no difference in arithmetic, if the multiplier and multiplicand are interchanged. If the same principle holds in algebra we may say that a positive number multiplied by a negative number is a negative number. This argument is given in some form by a number of our authors. There is another way by which we may approach this case. Suppose we take the series

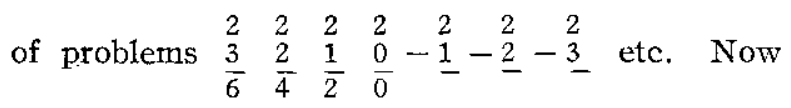

suppose that in one line representing positive and negative number, we represent in order the first four products and ask the pupils what they would expect the fifth product to be, the sixth, etc. Your pupils will give you the correct results, and if they have come to look on $0,-1,-2$, etc., as a continuation of feal number down from a starting point, they will feel that a positive number multiplied by a negative number is negative. And this agrees with the result obtained by the first method. Considering the question from the standpoint of the above or some similar series may seem novel, but is it not similar to the method of finding the value of $(\mathrm{I}+) \mathrm{x}$ when $\mathrm{x}$ is infinite? If we make $\mathrm{x}$ absolutely infinite we can do nothing with our function, but we attack the problem by finding the value which the function approaches as $x$ approaches infinity and we call that the value of the function when $x$ is infinite. I speak at length on this case as it is the most difficult with which we have to deal in any of the laws of signs. And I am just as willing as anyone to acknowledge that the above treatment is not satisfactory from the standpoint of technical reasoning, but $I$ am not willing to give up the above methods until some one will suggest one that is better.

The fourth case may be illustrated by problems in the form $9-2$ multiplied by -4 . -4 times $9=36,-4$ times -2 is numerically 8 , put in a question mark for the sign of the 8 , then make the expression equal to -4 times 7 , that is, -28 . Then let the pupils tell you what signe must replace the question mark in order to make the equation true. In this wya it is possible to force the pupil to tell you that a negative number multiplied by a nega- 
tive number is a positive number. If he is a thinking pupil he will be surprised but so were you the first time you came to this result.

Some pupils may be helped by a series of problems in which the multiplicand is a constant negative number and the multipliers decrease by ones from say 4 to -4 . The teacher who wishes to examine in detail the illustrations given by different authors for multiplication will not overlook those given by Aley \& Rothrock, also by Beman \& Smith. They are too long to quote here.

The signs in the four cases of division are readily determined by the pupil if he uses the same test for correct division that he he has used in arithmetic, namely, that the product of the divisor and quotent must equal the dividend.

The teacher will spend several recitations on the subject of this paper, and it will therefore be possible in class work to multiply illustrations. When the pupil is satisfied of the truth of any law of signs he should be compelled to commit the law. Take the law for subtraction for instance. If time is to be saved and the pupil to be in a condition to be helped by the teacher's suggestions, he must be able to give instantly the rule. In making the above suggestions I am not unconscious that they are arithmetical and inductive. Just as the proof given in arithmetic that the area of a rectangle is equal to the product of its base and altitude, is inductive, probably does not include fractions and is surely not such a proof as would be accepted in geometry and yet it does satisfy the mind as well as the latter proof. So the above proofs of the laws of signs are rather illustrations than proofs and are subject to many criticisms and yet, I for one, believe that they are proofs and that they will satisfy the pupils.

If we examine the subject of negative number as presented in the text-books of today we will find that the authors arrange themselves into two classes. In general the more elementary books attempt to prove these laws. Others represented by Chrystal lay down certain laws as fundamental in. algebra, being careful that the laws be self-consistent and that they include as particular cases the laws of arithmetic. Then the operations are defined by the laws. That is the law of signs, for example, help to determine the meaning of multiplication as that subject is expanded beyond its meaning in arithmetic. These authors go 
from the laws to the meaning of the operations and from the laws and operations to the applications. Let me quote from Chrystal: "It may be well to insist once more upon the exact position which they (the fundamental laws) hold in the science. To speak as is sometimes done of the proof of these laws in all their generality is an abuse of terms. They are simply laid down as the canons of the science. The best evidence that this is their real position is the fact that algebras are in use whose fundamental laws differ from those of ordinary algebra." In support of this he gives as an example the fact that in Quaternious $a b=b a$.

This confusion results from the following facts, the fundamental operations are very difficult of definition. And the definitions of discrete arithmetic are not satisfactory when we come to extending the number system. $4 \times 5$ is a matter of definition so far as the use of the word times is concerned, but if the number system is agreed upon and the word times is understood then problem becomes a matter of calculation. So if we start with certain notions as to how we shall apply the four fundamental operations in algebra, then the results are matters of calculation and demonstration not matters of definition.

There is always a chance that the order of the historical de:velopment of a subject is the pedagogical one. Diaphantes knew that "minus into minus gives plus" two thousand years before the process was reversed and "minus into minus gives plus" was made a part of the definition of multiplication. But what shall we say of the case cited by Chrystal Quaternious?

Again an historical incident helps us out. Sir William Hamilton did not start with the law that $a b=-$ ba and develop the subject but he started with certain conceptions and interpretations and after long struggles found that as he was representing and interpreting quantities and operations $a b$ ba as he had up to that time supposed.

Therefore I believe that with beginners we are right when we explain how we interpret negative number, and how we apply the fundamental operations and then make the laws of signs matters of demonstration. If the writers for advanced students can be more logical by reversing the process we surely have no objection. 